Universidad Nacional Experimental Francisco de Miranda (UNEFM). Santa Ana de Coro. Venezuela

Diana Alexandra Patiño-Quizhpi; Darwin Gabriel García-Herrera; María Isabel Álvarez-Lozano; Juan Carlos Erazo-Álvarez

DOI $10.35381 / \mathrm{cm} \cdot \mathrm{v} 6 \mathrm{i} 3.408$

\title{
Estrategias lúdicas para desarrollar la lecto-escritura mediante la plataforma Liveworksheets
}

\section{Ludic strategies will be applied to develop literary skills by using the platform} Liveworksheets.

\author{
Diana Alexandra Patiño-Quizhpi \\ diana.patino.27@est.ucacue.edu.ec \\ Universidad Católica de Cuenca, Azogues \\ Ecuador \\ https://orcid.org/0000-0002-5183-2961 \\ Darwin Gabriel García-Herrera \\ dggarciah@ucacue.edu.ec \\ Universidad Católica de Cuenca, Azogues \\ Ecuador \\ https://orcid.org/0000-0001-6813-8100 \\ María Isabel Álvarez-Lozano \\ mialvarezl@ucacue.edu.ec \\ Universidad Católica de Cuenca, Biblián \\ Ecuador \\ https://orcid.org/0000-0001-8029-1933 \\ Juan Carlos Erazo-Álvarez \\ jcerazo@ucacue.edu.ec \\ Universidad Católica de Cuenca, Cuenca \\ Ecuador \\ https://orcid.org/0000-0001-6480-2270
}

Recibido: 20 de agosto de 2020

Aprobado: 15 de noviembre de 2020 
Universidad Nacional Experimental Francisco de Miranda (UNEFM). Santa Ana de Coro. Venezuela

Diana Alexandra Patiño-Quizhpi; Darwin Gabriel García-Herrera; María Isabel Álvarez-Lozano; Juan Carlos Erazo-Álvarez

\title{
RESUMEN
}

La investigación tuvo por objeto determinar como el uso de la plataforma Liveworksheets permitió desarrollar las destrezas de lectura y escritura en estudiantes de cuarto grado de la Unidad Educativa "Luis Cordero" de la ciudad de Azogues. Es un estudio experimental con enfoque cuantitativo, realizado en dos intervenciones pre - test y post - test a través de la técnica de la observación con el instrumento ficha de observación. Los resultados obtenidos manifiestan cambios importantes en el desarrollo de la destreza de lectoescritura en la asignatura Lengua y Literatura, lo que es corroborado en el análisis realizado con el instrumento estadístico SPSS que lleva a verificar la validez de la hipótesis planteada al inicio a través de la prueba T-Student, confirmando que el uso de la plataforma mejora el desarrollo de una destreza importante en el proceso de enseñanza para los niños y niñas en sus primeros años de educación.

Descriptores: Tecnología educacional; juego educativo; material didáctico. (Palabras tomadas del Tesauro UNESCO).

\begin{abstract}
The objective of the research was to determine how the use of the Liveworksheets platform allowed the development of reading and writing skills in fourth-grade students from the "Luis Cordero" Educational Unit in the city of Azogues. It is an experimental study with a quantitative approach, carried out in two pre - test and post - test interventions through the technique of observation with the observation sheet instrument. The results obtained show important changes in the development of literacy skills in the Language and Literature subject, which is corroborated in the analysis carried out with the SPSS statistical instrument that leads to verify the validity of the hypothesis raised at the beginning through the T-Student test, confirming that the use of the platform improves the development of an important skill in the teaching process for boys and girls in their first years of education.
\end{abstract}

Descriptors: Educational technology; educational games; teaching materials. (Words taken from the UNESCO Thesaurus). 
Universidad Nacional Experimental Francisco de Miranda (UNEFM). Santa Ana de Coro. Venezuela

Diana Alexandra Patiño-Quizhpi; Darwin Gabriel García-Herrera; María Isabel Álvarez-Lozano; Juan Carlos Erazo-Álvarez

\section{INTRODUCCIÓN}

Desde los primeros niveles de educación en los que los infantes tienen gran curiosidad por aprender, es menester subrayar la gran importancia que tiene la asignatura de Lengua y Literatura en el proceso educativo, es importante que los niños aprendan a leer, escribir para mejorar su proceso educativo, pero justamente el desarrollo de estas destrezas han presentado desde tiempos atrás hasta la actualidad serios problemas en el ámbito educativo, es necesario cambiar las técnicas y las estrategias metodológicas, para alcanzar aprendizajes significativos desde los primeros niveles de enseñanza es cuando se debe aprovechar la predisposición e interés de los niños y niñas por aprender.

En una ficha informativa de la (Unesco [UNESCO], 2017), un porcentaje elevado de niños y adolescentes no alcanzan los mínimos requeridos en lectura y matemáticas de acuerdo a las proyecciones del Instituto de Estadística de la UNESCO (UIS) por sus siglas en inglés, lo que puede determinar un retroceso en el desarrollo de objetivos educacionales; de las cifras logradas por este instituto se ha establecido que más del 50\% corresponde a niños de educación primaria que no logran completar las competencias para leer, escribir y realizar cálculos matemáticos los datos encontrados, subrayan la necesidad de mejorar el acceso al desarrollo educativo.

En relación al tema a nivel del Ecuador (Loza, 2018), los procesos de lectura y escritura constituyen practicas o destrezas que marcan la vida del niño por lo que tienen gran importancia el aprendizaje basado en ellas se convierte en una capacidad de las personas que se debe ir construyendo desde los niveles más elementales sin embargo, existen grados de dificultad en la enseñanza por el estilo de aprendizaje que tiene cada estudiante lo que no favorece la aplicación de estrategias tradicionales estandarizadas, por el contrario, se debe recurrir a nuevas metodologías que ayuden en la adquisición de estas destrezas que son fundamentales en el desarrollo educativo de las personas. 
Universidad Nacional Experimental Francisco de Miranda (UNEFM). Santa Ana de Coro. Venezuela

Diana Alexandra Patiño-Quizhpi; Darwin Gabriel García-Herrera; María Isabel Álvarez-Lozano; Juan Carlos Erazo-Álvarez

En las instituciones de la ciudad de Azogues, en los grados de básica elemental se han determinado procesos educativos de cohorte tradicional que pueden parecer adecuados en el nivel educativo en el que se utilicen, pero que no representan un aprendizaje significativo, ya que algunos niños presentan dificultades en el momento de leer como; silabeo, lectura por renglones marcados, confusión en el orden de letras o fonemas, de igual manera escriben con trazos ilegibles y en desorden; entre algunas deficiencias la destreza de lectoescritura, lo que exige cambios en el proceso académico con la implementación de técnicas interactivas lúdicas.

La necesidad de fomentar las destrezas lectoras y la lectura es muy recurrente ante esto (Suarez, Pérez, Vergara, \& Alferez, 2015), mencionan que en Latinoamérica los procesos de lectoescritura siguen ligados al aprendizaje tradicional sin tomar en consideración las nuevas estrategias metodológicas, a través del uso de tecnologías innovadoras que tengan repercusión en el ámbito extracurricular de hecho del estudio realizado por ellos se pudo establecer que la inclusión de herramientas tecnológicas mejora sustancialmente el desarrollo de la destreza de lectoescritura, lo que afianza otras competencias en la vida escolar y personal.

Coincidiendo con lo afirmado, es importante señalar que por la situación de emergencia sanitaria que vive el país y aún después de ella las metodologías innovadoras han abierto paso en el ámbito educativo con el uso de herramientas tecnológicas que se vuelve cotidiano y sobre todo de gran utilidad, los niños y niñas puedan interactuar de mejor manera a través de estos medios, que por otra parte posibilitan la actividad lúdica para facilitar el proceso educativo y la adquisición de destrezas es oportuno mencionar que la plataforma Liveworksheets, tiene todas las características para el desarrollo de las destrezas de lectura y escritura en los niños y niñas.

Esta plataforma interactiva cuenta con muchas variaciones, no solamente para el área de Lengua y Literatura, también para otras asignaturas se basa en la diagramación de hojas digitales interactivas con las que se consolidan los aprendizajes, logrando 
Universidad Nacional Experimental Francisco de Miranda (UNEFM). Santa Ana de Coro. Venezuela

Diana Alexandra Patiño-Quizhpi; Darwin Gabriel García-Herrera; María Isabel Álvarez-Lozano; Juan Carlos Erazo-Álvarez

interesarle al niño que en esta actividad lúdica aprende jugando y juega para aprender, a la vez que tiene la posibilidad de retroalimentar sus conocimientos a través de videos, colores, dibujos, diagramas y tareas virtuales, los estudiantes razonan y construyen nuevos conocimientos, pero sobre todo desarrollan la lectura y escritura que es básico para poder realizar sus actividades en todas las asignaturas.

Por lo descrito se busca determinar cómo el uso de la plataforma Liveworksheets contribuye en el desarrollo de las destrezas de lectura y escritura en estudiantes de los niveles educativos elementales, tomando como referente la aplicación de esta estrategia tecnológica con estudiantes de cuarto grado, para esclarecer los siguientes cuestionamientos: ¿Es de aporte significativo la aplicación de metodologías innovadoras? ¿La plataforma Liveworksheets mejorará el proceso de enseñanza aprendizaje en el desarrollo de las destrezas de lectoescritura? Con los posibles resultados se espera probar la hipótesis: "A mayor uso de la plataforma Liveworksheets mejor aprendizaje en el área de Lengua y Literatura".

\section{Referencial teórico}

El aprendizaje cada día requiere de transformación en cuanto a su modelo educativo, pasar de una educación conductista a una que ayude a los estudiantes a construir su propio conocimiento precisamente desde las primeras fases del aprendizaje tomando como fundamento una de las asignaturas que más interrelacionada está con las demás en la malla curricular como es Lengua y Literatura.

Es necesario buscar mejores alternativas para desarrollar destrezas que permitan la estructuración correcta del conocimiento. La educación de corte tradicionalista que se mantiene todavía con sus técnicas y métodos conductistas, se limitan al dictado aburrido, al uso exclusivo de la pizarra, desmotivando a los niños y niñas, se limita la participación activa y el proceso se vuelve monótono, produciendo desinterés en el aprendizaje. Es el momento de repensar los procesos de materias importantes como Lengua y Literatura 
Universidad Nacional Experimental Francisco de Miranda (UNEFM). Santa Ana de Coro. Venezuela

Diana Alexandra Patiño-Quizhpi; Darwin Gabriel García-Herrera; María Isabel Álvarez-Lozano; Juan Carlos Erazo-Álvarez

con la prioridad de utilizar estrategias y metodologías activas que permitan volver sugestiva y motivadora la enseñanza por parte de los docentes que con capacitación y preparación en el uso de herramientas tecnológicas traten de innovar el desarrollo de las destrezas.

Para fundamentar la investigación hay estudios anteriores realizados a nivel local, nacional e internacional que confirman algunos aspectos de lo que se quiere lograr con la propuesta de este artículo. Algunos autores emiten sus ideas desde diferentes perspectivas, realizan propuestas, establecen conclusiones, en algunos casos positivos y en otros negativos, que sirven para poder orientar la investigación.

En un informe de la (FOUNDATION LEGO [UNICEF], 2018), se menciona la necesidad de explicar los factores por los que en muchos países, no se han integrado dentro del sistema educativo los juegos para el aprendizaje, señalando entre ellos: incapacidad de apreciar el valor del juego como base en la adquisición de nuevos conocimientos, se privilegia el estilo memorístico, a esto se suman las ideas equivocadas por parte de los padres de familia acerca del juego, lo ven como algo sin trascendencia y que quitan tiempo al verdadero proceso de aprendizaje.

De acuerdo con este estudio otro aspecto que debe ser tomado en cuenta es el de las mallas curriculares y los modelos educativos, ya que no incluyen el juego en ninguna de las asignaturas esto ocurre en la mayor parte de países evaluados por Unicef, de igual manera hay una falta de capacitación en los docentes en lo que se relaciona con las actividades lúdicas para el proceso educativo, así como la presencia numerosa de estudiantes en las aulas, que limitan la posibilidad de utilizar el juego como estrategia, con base a estas falencias las autoridades y docentes van comprendiendo la necesidad de implementar y usar estrategias lúdicas para mejorar la enseñanza.

Igualmente en Colombia se realiza un estudio sobre la lúdica como estrategia para favorecer el proceso de aprendizaje en niños de básica elemental, el mismo que de acuerdo a (Calderón, Marín, \& Vargas, 2014), se menciona que de la observación a la 
Universidad Nacional Experimental Francisco de Miranda (UNEFM). Santa Ana de Coro. Venezuela

Diana Alexandra Patiño-Quizhpi; Darwin Gabriel García-Herrera; María Isabel Álvarez-Lozano; Juan Carlos Erazo-Álvarez

comunidad educativa se determina que es necesario realizar una actividad innovadora a través del proyecto pedagógico de aula: el gozo del saber, realizan actividades relacionadas a los intereses, necesidades y niveles educativos para fortalecer el aprendizaje. El proceso de interacción cuenta con la colaboración de padres de familia, docentes y estudiantes logrando un resultado satisfactorio y de mucha utilidad para la enseñanza ya que se desarrollan las habilidades en los niños y niñas para una mayor comunicación participativa.

En el Ecuador, (Chicaiza, 2015) realiza la propuesta de diseño de una estrategia lúdica para mejorar la comprensión lectora, fundamentando su proyecto en el descubrimiento de la dificultad que experimentan la mayoría de los estudiantes tienen dificultad para comprender y entender textos básicos, el aprendizaje es memorístico y se transforman en receptores de información sin discernimiento, por otra parte los docentes no tienen estrategias que motiven la lectura como un medio de adquirir nuevos conocimientos, de ahí que nace la necesidad de diseñar una táctica lúdica eficiente para poder desarrollar la destreza de lectura, que posibilita a mejorar la educación.

También, (Sibri, 2019), hace un estudio con estudiantes del cuarto grado de básica en Ecuador en el que observa un bajo rendimiento académico en la materia de Lengua y Literatura, debido a la displicencia de los docentes que no utilizan en sus clases estrategias metodológicas que les permitan motivar en los estudiantes el interés para adquirir los conocimientos necesarios en esta importante asignatura, anota además que no hay capacitación en los maestros para que se busquen soluciones. Entre estas se pueden realizar la capacitación de los docentes en la aplicación de estrategias didácticas a través de los juegos, para que los educandos puedan alcanzar un mejor rendimiento. Dentro de los nuevos parámetros presentes en el modelo educativo que maneja el Ministerio de Educación del Ecuador (Ministerio de Educación del Ecuador [MINEDUC], 2020), por la situación que se tiene en el país se pide ir hacia un proceso educativo más activo y que permita construir el conocimiento de mejor manera que los docentes se 
Universidad Nacional Experimental Francisco de Miranda (UNEFM). Santa Ana de Coro. Venezuela

Diana Alexandra Patiño-Quizhpi; Darwin Gabriel García-Herrera; María Isabel Álvarez-Lozano; Juan Carlos Erazo-Álvarez

vuelvan motivadores hacia nuevas formas de aprender, los estudiantes respondan positivamente a las estrategias y los padres de familia colaboren en este proceso, motivar a los docentes a capacitarse para el manejo de metodologías activas en el desarrollo de destrezas como la lectoescritura.

Las metodologías activas aplicada a la educación son herramientas eficaces que desarrollan destrezas importantes en cada una de las asignaturas, en el caso de Lengua y Literatura es de bastante utilidad el uso de la Plataforma digital Liveworksheets que sirve para la creación de libros de trabajo, registros de alumnos, elaborar fichas de trabajo interactivas que pueden ser contestadas y evaluadas por el estudiante, despierta su curiosidad y trata de comprometerse en realizar las tareas que el docente sube en la plataforma, al ser interactiva no se requiere imprimir ya que el sitio da la posibilidad de calificar y guardar las tareas que se han evaluado.

En esta plataforma se presentan muchas aplicaciones atractivas ya que las tarjetas pueden contener sonidos, videos y múltiples actividades que son de atracción para los niños sobre todo, esta plataforma ha ganado connotación en estos tiempos en los que las nuevas tecnologías están cada vez más incursionando en la educación, permitiendo un universo amplio de estrategias didácticas al servicio de los docentes y de los estudiantes que se sienten motivados por utilizar estas plataformas recordando que son producto de una nueva era, la era digital.

\section{Plataforma Liveworksheets}

Referente a las aplicaciones tecnológicas en la educación es interesante revisar lo que presentan (Torres \& Cobo, 2017), en su estudio que busca explicar el papel de las tecnologías en el proceso de la enseñanza, subrayando la importancia de su función al materializar las finalidades más trascendentales de la educación en los momentos actuales y que apuntan a la formación integral de los seres humanos, así como del modelo de sociedad en la que se desarrollen; en este sentido se evidencia que los 
Universidad Nacional Experimental Francisco de Miranda (UNEFM). Santa Ana de Coro. Venezuela

Diana Alexandra Patiño-Quizhpi; Darwin Gabriel García-Herrera; María Isabel Álvarez-Lozano; Juan Carlos Erazo-Álvarez

actores, estudiantes y docentes asumen sus roles y conocen su situación frente al proceso que exige la sociedad en su formación ya que el objetivo primordial de la educación es el desarrollo de la personalidad.

De igual forma acerca de las nuevas tecnologías (Domingo \& Fuentes, 2019), sostienen que en la actualidad la información digital llega a todos los lugares de diferentes maneras todos los estudiantes saben manejar muy bien las herramientas tecnológicas debido a la curiosidad e interés que generan en ellos, esto ayuda en el proceso educativo al momento en que los docentes desarrollen un entorno de aprendizaje que favorezca la enseñanza, por medio de la aplicación de la tecnología que favorecen la adquisición de nuevos conocimientos, en el estudio llegan a conclusiones que se pueden resumir en la aceptación por parte de los docentes, ya que un buen proceso educativo requiere el uso de estrategias didácticas basadas en las TIC.

Entre las aplicaciones educativas que se pueden observar en la web hay algunas que se pueden usar en Lengua y Literatura destacando para esta investigación la plataforma Liveworksheets, que tiene que ver con las hojas de trabajo virtuales y todo lo relacionado a sus fundamentos, características, ventajas y desventajas dentro del proceso educativo, los entornos en los que se puede aplicar y la ductilidad que tiene para ser un aporte en el desarrollo de destrezas como la lectoescritura en Lengua y Literatura.

En relación a la descripción de la plataforma (Del-Prete \& Cabero-Almenara), esbozan algunas particularidades mencionan que su principal característica, es la de transformar hojas tradicionales de trabajo presentadas en pdf, jpg, doc, etc., en hojas interactivas con autocorrección, incluyendo multimedia en videos, sonidos, imágenes entre otras, cabe indicar que es una plataforma gratuita y que puede ser utilizada por los docentes para enviar tareas a sus estudiantes pero se limita a cien estudiantes, por lo que un docente debe crearse otra cuenta para poder interactuar con otros grupos. La plataforma Liveworksheets surge para ir cubriendo algunas necesidades de docentes que tienen asignaturas relacionadas con los diferentes idiomas. 
Universidad Nacional Experimental Francisco de Miranda (UNEFM). Santa Ana de Coro. Venezuela

Diana Alexandra Patiño-Quizhpi; Darwin Gabriel García-Herrera; María Isabel Álvarez-Lozano; Juan Carlos Erazo-Álvarez

En el caso de los países de habla hispana esta estrategia se emplea en los diferentes niveles educativos en el área de Lengua y Literatura Española, con lo que se puede afirmar que es de gran ayuda para la realización de tareas de una manera interactiva. Las características de esta plataforma la hacen muy atractiva para utilizarla en el proceso de enseñanza de materias que requieren de procesos didácticos interactivos para su mejor comprensión, sus ventajas son muy grandes la adquisición del conocimiento a través del juego con actividades lúdicas relacionadas con los temas de clase dan lugar a mejorar el aprendizaje.

Con el uso de la Plataforma se obtienen excelentes resultados con porcentajes altos de motivación en los niños y niñas al actuar interactivamente, al tratarse de un entorno virtual que posee tecnología web 2.0. Es necesario recalcar que el uso de las herramientas informáticas se adapta de acuerdo a las necesidades de cada asignatura, en el caso concreto de Lengua y Literatura, genera beneficios para desarrollar la destreza de lectoescritura en niños de básica elemental para lo que debe existir el compromiso, la perseverancia y la capacitación de los docentes para innovar sus clases partiendo del aprendizaje lúdico que es indispensable en el proceso educativo.

\section{La Lectoescritura}

En el área de Lengua y Literatura la destreza que genera mejores resultados en el aprendizaje es la lectoescritura, ya que se interrelaciona con las demás tanto en Lenguaje como en las otras asignaturas, el proceso de aprender a leer y escribir, se inicia desde tempranas edades y en los primeros niveles educativos básicamente se debe desarrollar a través de estrategias que logren preparar el proceso de comunicación entre los niños y su entorno, con sus compañeros, con su docente y con su familia.

Los seres humanos buscan comunicarse a través de cualquier medio en este aspecto según menciona (Niland, 2007), lo han hecho desde las artes, con la narración de cuentos, la música, la danza y combinando diferentes formas para expresar sus ideas, 
Universidad Nacional Experimental Francisco de Miranda (UNEFM). Santa Ana de Coro. Venezuela

Diana Alexandra Patiño-Quizhpi; Darwin Gabriel García-Herrera; María Isabel Álvarez-Lozano; Juan Carlos Erazo-Álvarez

en este aspecto los docentes a través de la lectura de artículos que combinen con artes como la música y la danza, se cree que es posible desarrollar los hábitos de escritura proporcionando a los niños una experiencia lúdica a través de las artes le da la importancia al aprendizaje de la lectura con estrategias que se basan en otras áreas, interrelacionadas con el juego y las artes.

(Ferrandi, 2016), le confiere importancia a la lectoescritura cuando indica que la práctica de esta destreza confiere al ser humano su identidad con el mundo, a través de la comunicación que se fortalece al leer y escribir descubre su entorno natural y social. Más allá de que las técnicas y tecnologías muchas veces obedecen a la orientación de los gobiernos para su difusión, en los actuales tiempos al haberse desarrollado y estar al alcance de todos se debe proyectar desde niños el interés que los permitan tener criterios de formación personal y autónoma para que pueda construir con equidad su ambiente socio-cultural.

En América Latina y el Caribe el (INSTITUTO AMERICANO DE INVESTIGACION [AIRUSAID], 2020), ha diseñado el programa de Capacidad de lectura de América Latina y el Caribe (LCRP), puesto que el aprendizaje es una habilidad fundamental para toda persona para el desarrollo social y económico de un país, por lo que este programa tiene como meta aumentar el impacto, los niveles y la sostenibilidad de la destreza de lectura en los países de esta región, coloca sus esfuerzos para impulsar el aprendizaje de lectura de los primeros grados, en particular para los niños desfavorecidos, a través de la formación de docentes capacitados para lograr el desarrollo de esta destreza. Para poder lograr este objetivo AIR y sus socios estratégicos en cada país recopilan información en investigaciones de alta calidad la que es proporcionada a los actores principales, autoridades y docentes a fin de capacitar en el desarrollo de la destreza de lectura por considerarla fundamental en los procesos académicos.

De lo aquí expuesto, se denota la importancia de desarrollar correctamente la destreza leer y escribir en los primeros años de educación, ya que se proyecta en la vida estudiantil 
Universidad Nacional Experimental Francisco de Miranda (UNEFM). Santa Ana de Coro. Venezuela

Diana Alexandra Patiño-Quizhpi; Darwin Gabriel García-Herrera; María Isabel Álvarez-Lozano; Juan Carlos Erazo-Álvarez

de cada persona puntualizando que la lectoescritura facilita los procesos educativos en las diferentes áreas del conocimiento, al leer correctamente los niños y niñas podrán ser capaces de tener criticidad y autonomía al momento de emitir sus comentarios y aportes en la construcción del saber. Esto requiere de la capacitación de los docentes en el uso de estrategias lúdicas que permitan llegar hacia los estudiantes con prácticas innovadoras que desarrollen esta importante destreza.

\section{METODOLOGÍA}

La investigación se fundamentó en un diseño experimental, con enfoque cuantitativo y alcance descriptivo, de cohorte longitudinal, que se desarrolló en dos fases de intervención: el primer período ( $1^{\circ}$ clase) sin aplicar estrategias lúdicas y el segundo período ( $2^{\circ}$ clase) con el uso de la Plataforma Liveworksheets, aplicando la técnica de observación y como instrumento la ficha de observación, con 12 ítems, que corresponden a los aspectos que se querían observar, la población estuvo constituida por 36 estudiantes del paralelo A del cuarto grado de Educación General Básica Elemental de la Unidad Educativa "Luis Cordero" de la ciudad de Azogues, Provincia del Cañar, matriculados para el año lectivo 2020 - 2021.

La observación directa desde los aportes de (Hernández, Fernández, \& Baptista, 2014) es el registro organizado que tiene validez y seguridad, al mirar comportamientos 0 conductas manifiestan en donde el observador puede obtener datos en forma directa y presencial. La técnica se desarrolló en dos etapas o períodos, la primera clase denominada pre-test con una duración de 40 minutos en la que se utilizó material concreto y de manera tradicional; y la segunda clase o pos-test con un período de 40 minutos en la que se empleó la Plataforma Liveworksheets.

Para registrar los resultados se utilizó una ficha de observación previamente diseñada con 12 ítems de acuerdo a las actividades planificadas: Actividad, entusiasmo, interacción, tareas, relación, interés, motivación, uso de plataforma, aprendizaje, 
Universidad Nacional Experimental Francisco de Miranda (UNEFM). Santa Ana de Coro. Venezuela

Diana Alexandra Patiño-Quizhpi; Darwin Gabriel García-Herrera; María Isabel Álvarez-Lozano; Juan Carlos Erazo-Álvarez

preferencia, destreza y reacción, con parámetros de valoración 1: nunca, 2: rara vez, 3: frecuentemente, 4 casi siempre, 5 siempre.

El instrumento fue validado a través del cálculo de fiabilidad del coeficiente del Alfa de Cronbach igual a 0.943 con lo que se validó 12 elementos. Los datos recopilados se procesaron en Excel y luego en el instrumento estadístico SPSS en el que se establecen estadísticos comparativos.

\section{RESULTADOS}

Para probar la validez de la hipótesis se usó el T- Student que permitió verificar que a mayor uso de Liveworksheets se mejora la destreza de lectoescritura en niños de básica elemental, estableciendo un $\mathrm{p}$-valor de a $\leq 0,05$ para que sea válida la hipótesis, o un $\mathrm{p}$ - valor de a $\geq 0,05$ para que se considere nula la hipótesis.

A continuación, se realiza un análisis de los resultados globales obtenidos en el estudio.

Tabla 1.

Resultados Globales Pre - test y Post - test .

\begin{tabular}{|c|c|c|c|c|c|}
\hline Variable & & $\mathrm{N}$ & Media & $\begin{array}{c}\text { Desv. } \\
\text { Desviación }\end{array}$ & $\begin{array}{l}\text { Desv. Error } \\
\text { promedio }\end{array}$ \\
\hline \multirow{2}{*}{$\begin{array}{l}\text { Despierta su interés por los recursos interactivos } \\
\text { que presenta la Plataforma } \\
\text { Liveworksheets. }\end{array}$} & Pre - test & 72 & 2,74 & 0,650 & 0,077 \\
\hline & Post - test & 72 & 4,93 & 0,256 & 0,030 \\
\hline \multirow{2}{*}{ Desean utilizar en su aprendizaje la Plataforma } & Pre - test & 72 & 2,92 & 0,575 & 0,068 \\
\hline & Post - test & 72 & 4,93 & 0,256 & 0,030 \\
\hline \multirow{2}{*}{$\begin{array}{l}\text { Les interesa y motiva el uso de Liveworksheets } \\
\text { en la clase. }\end{array}$} & Pre - test & 72 & 1,25 & 0,524 & 0,062 \\
\hline & Post - test & 72 & 4,90 & 0,298 & 0,035 \\
\hline \multirow{2}{*}{ Ha mejorado su destreza de lecto escritura } & Pre - test & 72 & 2,53 & 0,731 & 0,086 \\
\hline & Post - test & 72 & 4,57 & 0,624 & 0,074 \\
\hline
\end{tabular}

Fuente. Elaboración propia. 
Universidad Nacional Experimental Francisco de Miranda (UNEFM). Santa Ana de Coro. Venezuela

Diana Alexandra Patiño-Quizhpi; Darwin Gabriel García-Herrera; María Isabel Álvarez-Lozano; Juan Carlos Erazo-Álvarez

En referencia al interés que despierta en los niños y niñas la plataforma en el pre - test se observa una media de 2,74 y en el post - test de 4,93 , con estos datos se evidencia que existe un notable interés en los recursos interactivos que presenta la plataforma en la realización de sus tareas, lo que ofrece cambios significativos que se confirman con el índice de la prueba T que es de $(0,000)$, de igual manera el deseo de utilizar la plataforma en el pre - test alcanza una media de 2,92, mientras que en post - test alcanza una media de 4,93 que se verifica con un índice en la prueba $T$ de $(0,000)$.

En lo que se refiere a la motivación e interés por la plataforma en el pre - test la media alcanzada es de 1,25 y la media del post - test es de 4,90, alcanzando un índice de $(0,000)$ en la prueba $T$, lo que demuestra que existe interés en el empleo de Liveworksheets en el aula y fuera de ella en las tareas, en cuanto a la destreza de lectoescritura se tiene una media de 2,53 en el pre - test y 4,47 en el post - test, avalado por un índice de $(0,000)$ en la prueba $T$, que permite evidenciar que el uso adecuado de la plataforma favorece la lectura y la escritura en niños y niñas de básica elemental.

Los resultados expuestos indican que la implementación y el uso de la plataforma Liveworksheets es bueno para mejorar la lectoescritura, fundamental en el proceso educativo y que contribuye en el aprendizaje de otras asignaturas relacionadas con Lengua y Literatura, así lo considera (Caravajal, 2020), al coincidir en la actualidad la práctica de esta destreza posibilita muchas oportunidades que se relacionan con nuevas estrategias, técnicas, recursos y métodos que posibilitan el uso de herramientas tecnológicas, está acorde a la era digital que provoca un avance significativo en la adquisición de nuevos conocimientos en estudiantes ávidos de explorar la web.

Por otra parte la plataforma Liveworksheets proporciona variados recursos interactivos que la hacen fácil de usar con una utilidad pedagógica importante, esta plataforma promueve la interactividad a través de hojas de trabajo virtuales que tiene una versatilidad que favorece a los procesos de enseñanza, al mismo tiempo que permiten la retroalimentación de los conocimientos y los estudiantes mientras juegan pueden 
Universidad Nacional Experimental Francisco de Miranda (UNEFM). Santa Ana de Coro. Venezuela

Diana Alexandra Patiño-Quizhpi; Darwin Gabriel García-Herrera; María Isabel Álvarez-Lozano; Juan Carlos Erazo-Álvarez

aprender por la manera en que se manejan las actividades virtuales entre los docentes con los estudiantes, ya que se motivan y destacan su creatividad en esta plataforma.

\section{PROPUESTA}

El juego es parte de la naturaleza de los seres humanos, desarrollándose desde tempranas edades y en todo el entorno de los niños y niñas mismo que está inmerso en el ámbito educativo, es la principal actividad que favorece su integración social por lo que la propuesta está orientada a la implementación de destrezas lúdicas que favorezcan al desarrollo de la destreza de lectoescritura, a través de la plataforma Liveworksheets que con sus fichas interactivas favorece el aprendizaje dinámico y creativo.

La finalidad de la propuesta es la implementación de este recurso tecnológico en el nivel de básica elemental y su derivación hacia otros niveles, dentro del contexto educativo también es de mucha utilidad para los docentes puesto que al conocer la manera de interactuar en esta plataforma pueden crear numerosas tareas interactivas con videos, pruebas, dibujos, etc., de acuerdo a la necesidad educativa que tienen en sus materias. Se la implementó con los siguientes tópicos: objetivos, competencias, contenidos, secuencia, evaluación. En la tabla se colocan de manera resumida, pero luego se lo detalla, para que se pueda entender cómo se desarrolló la propuesta dentro de esta investigación. 
CIENCIAMATRIA

Revista Interdisciplinaria de Humanidades, Educación, Ciencia y Tecnología

Año VI. Vol. VI. N³. Edición Especial III. 2020

Hecho el depósito de ley: pp201602FA4721

ISSN-L: 2542-3029; ISSN: 2610-802X

Universidad Nacional Experimental Francisco de Miranda (UNEFM). Santa Ana de Coro. Venezuela

Diana Alexandra Patiño-Quizhpi; Darwin Gabriel García-Herrera; María Isabel Álvarez-Lozano; Juan Carlos Erazo-Álvarez

\section{Tabla 2.}

Propuesta para el desarrollo de la lectoescritura a través de Liveworksheets.

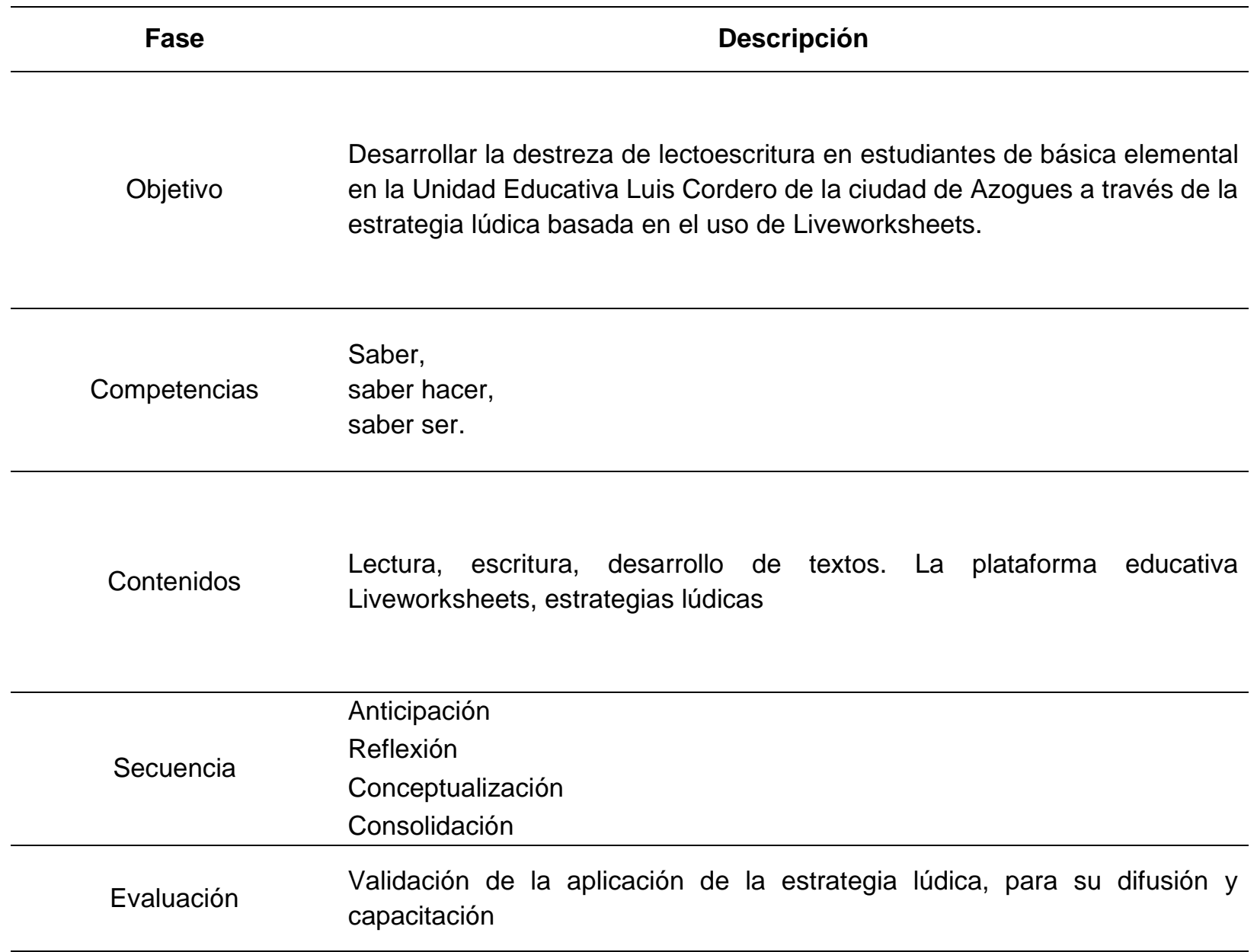

Fuente. Elaboración propia. 
Universidad Nacional Experimental Francisco de Miranda (UNEFM). Santa Ana de Coro. Venezuela

Diana Alexandra Patiño-Quizhpi; Darwin Gabriel García-Herrera; María Isabel Álvarez-Lozano; Juan Carlos Erazo-Álvarez

Objetivo: Con la aplicación de la estrategia lúdica por medio de la plataforma se quiere desarrollar la destreza de lecto escritura en niños y niñas del cuarto grado de básica elemental, para que se multiplique en los demás grados y niveles.

Competencias: Saber las actividades propuestas ayudan a fortalecer el conocimiento; saber hacer que son actividades, recursos y tareas para adquirir el desarrollo de destrezas; saber ser con actividades donde se fortalezcan valores como honestidad, respeto y se motive la creatividad y la participación.

Contenidos: Conceptos básicos y características de cada uno de los aspectos que se requieren explicar para la comprensión en la aplicación de la estrategia lúdica para el desarrollo de la destreza de lectoescritura.

Secuencia: Se siguen todos los pasos que se establece en el currículo educativo para insertar la clase en el objetivo que queremos alcanzar.

Evaluación: Al final del proceso desarrollado es conveniente la validación de la propuesta la misma que para lograr imparcialidad se efectúa por parte de actores externos y que son colegas del área de Lenguaje. Esto posibilita la difusión de la experiencia para que se la pueda retomar por otros docentes y en otras materias.

\section{CONCLUSIONES}

El área de Lengua y Literatura es fundamental para la adquisición de conocimiento en otras áreas de la educación, por lo que es sumamente importante poner bases sólidas en el proceso educativo de los estudiantes desde sus primeros años de vida; el desarrollo de destrezas como la lectura y la escritura en los niños y niñas de educación básica elemental tiene prioridad para poder conectarlas con las otras materias, si un niño lee correctamente puede interpretar problemas matemáticos, si escribe correctamente puede realizar tareas para diferentes ámbitos, entonces la lectoescritura debe ser trabajada con especial énfasis en los primeros años de escolaridad. 
Universidad Nacional Experimental Francisco de Miranda (UNEFM). Santa Ana de Coro. Venezuela

Diana Alexandra Patiño-Quizhpi; Darwin Gabriel García-Herrera; María Isabel Álvarez-Lozano; Juan Carlos Erazo-Álvarez

Aplicar en el contexto educativo estrategias lúdicas que contribuyan al desarrollo de destrezas en las diferentes asignaturas es una necesidad urgente de solucionar, el aprendizaje a través del juego es una metodología que está dando excelentes resultados en la actualidad, por ello es preciso que los docentes se capaciten en estos temas para poder realizar un trabajo pedagógico beneficioso para los estudiantes en todos los niveles educativos, las herramientas tecnológicas y la metodología innovadora son recursos que están al alcance de la mano de los docentes y los estudiantes solo basta con el compromiso que se ponga para que la educación consiga su objetivo final.

Las posibilidades que brinda la plataforma Liveworksheets no solo es la interacción entre docentes y estudiantes, es también la motivación para el aprendizaje en los niños y niñas, la conciencia del trabajo a través del juego que lleva a una interrelación social educativa en un intercambio que lo transporta a trabajar confiadamente, lo que resulta de beneficio para su formación integral, desarrollando destrezas importantes en su aprendizaje las que fluyen naturalmente en el entorno lúdico que la plataforma proporciona por su practicidad en el manejo de las fichas interactivas que vuelve atractivas las tareas y el conocimiento se desarrolla espontáneamente.

\section{REFERENCIAS CONSULTADAS}

Calderón, L., Marín, S., \& Vargas, N. (2014). La lúdica como estrategia para favorecer el Proceso de aprendizaje en niños de edad preescolar lbague [Play as a strategy to promote the learning process in preschool-age children lbague]. Ibagué: Universidad del Tolima. Obtenido de https://n9.cl/cbml

Caravajal, M. (2020). Técnicas Lúdicas Interactivas en Google Classroom para perfeccionar la lectoescritura en Básica Elemental.[Interactive Playful Techniques in Google Classroom to perfect basic elementary literacy.]. Universidad Tecnológica Israel, Escuela de Posgrados. Quito: UTI. Obtenido de https://n9.cl/9tta 
Universidad Nacional Experimental Francisco de Miranda (UNEFM). Santa Ana de Coro. Venezuela

Diana Alexandra Patiño-Quizhpi; Darwin Gabriel García-Herrera; María Isabel Álvarez-Lozano; Juan Carlos Erazo-Álvarez

Chicaiza, M. (2015). Diseño de una estategia lúdica para mjorar la comprensión lectora[Design of a playful strategy to improve reading comprehension]. Ambato: PUCE. Obtenido de https://n9.cl/ais6

Domingo, M., \& Fuentes, M. (2019). Innovación educativa: Experimentar con las Tic y reflexionar sobre su uso.[Educational innovation: Experimenting with ICT and reflecting on its use.]. Pixel-Bit. Revista de Medios y Educación, 0(36), 171 - 180. Obtenido de https://n9.cl/mc9ez

Ferrandi, L. (2016). La lectoescritura como un modo de construcción del mundo [Literacy as a way of building the world]. Letras, $0(5), 1-4$. Obtenido de https://n9.cl/wbsl

FOUNDATION LEGO [UNICEF]. (2018). Aprendizaje a través del juego. Obtenido de https://n9.cl/8r4n

Del-Prete, A, \& Cabero-Almenara, J. (2019). Las plataformas de formación virtual: algunas variables que determinan su utilización. Apertura (Guadalajara, Jal.), 11(2), 138-153. https://doi.org/10.32870/ap.v11n2.1521

Hernández, R., Fernández, C., \& Baptista, M. (2014). Metodología de la Investigación. México: MacGraw Hill Education. Recuperado el 20 de julio de 2020

INSTITUTO AMERICANO DE INVESTIGACION [AIR-USAID]. (2020). PROJECT:USAID/Latin America and Caribbean Reads Capacity Program (LRCP)[Proyecto de capacidad en lectura en AL y el Caribe]. USAID. Washington: AIR. Obtenido de https://n9.cl/vupr

Loza, J. (2018). Adaptaciones curriculares y el aprendizaje de lectoescritura en estudiantes de educación general básica elemental del Colegio Gutenberg Schule[Curricular adaptations and literacy learning in elementary general education students of the CollegeGutenberSch]. Universidad Andina Simón Bolivar. Quito: UASB. Obtenido de https://n9.cl/8cwg1

Ministerio de Educación del Ecuador [MINEDUC]. (2020). Curiculo priorizado 2020-2021. Quito: MINEDUC. Obtenido de https://n9.cl/cndot

Niland, A. (2007). Musical Stories: Strategies for Integrating Literature and Music for Young Children [Historias musicales: estrategias para integrar literatura y música para niños pequeños]. Australasian Journal of Early Childhood, 32(4), 7-11. doi:doi:10.1177/183693910703200403 
Universidad Nacional Experimental Francisco de Miranda (UNEFM). Santa Ana de Coro. Venezuela

Diana Alexandra Patiño-Quizhpi; Darwin Gabriel García-Herrera; María Isabel Álvarez-Lozano; Juan Carlos Erazo-Álvarez

Sibri, S. (2019). Bajo rendimiento académico en la materia de Lengua y Literatura en los estudiantes de Cuarto de EGB de la UE Buena Esperanza [Low academic performance in the subject of Language and Literature in the fourth grade students of EGB de la UE Buena Esperanza]. Cuenca: Universidad Politécnica Salesiana. Recuperado de https://www.redalyc.org/pdf/284/28437146008.pdf

Suarez, A., Pérez, C., Vergara, M., \& Alferez, V. (2015). Desarrollo de la lectoescritura mediante TIC y recursos educativos abiertos [Development of literacy through ICT and open educational resources]. Revista de Innovación EducativaApertura, 7(1), 1-7. Obtenido de https://n9.cl/fknh

Torres, P., \& Cobo, J. (2017). Tecnología educativa y su papel en el logro de los fines de la educación.[Educational technology and its role in achieving the aims of education]. Educere, 21(68), 31-40. Recuperado de https://n9.cl/nrcdx

Unesco [UNESCO]. (2017). Más de la Mitad de los Niños y Adolescentes en el Mundo No Está Aprendiendo. https://n9.cl/o9wr

@2020 por los autores. Este artículo es de acceso abierto y distribuido según los términos y condiciones de la licencia Creative Commons Atribución-NoComercial-Compartirlgual 4.0 Internacional (CC BY-NC-SA 4.0)

(https://creativecommons.org/licenses/by-nc-sa/4.0/) 